\title{
Secure Massive MIMO Transmission in the Presence of an Active Eavesdropper
}

\author{
Yongpeng Wu, Robert Schober, Derrick Wing Kwan Ng, Chengshan Xiao, and Giuseppe Caire
}

\begin{abstract}
In this paper, we investigate secure and reliable transmission strategies for multi-cell multi-user massive multipleinput multiple-output (MIMO) systems in the presence of an active eavesdropper. We consider a time-division duplex system where uplink training is required and an active eavesdropper can attack the training phase to cause pilot contamination at the transmitter. This forces the precoder used in the subsequent downlink transmission phase to implicitly beamform towards the eavesdropper, thus increasing its received signal power. We derive an asymptotic achievable secrecy rate for matched filter precoding and artificial noise (AN) generation at the transmitter when the number of transmit antennas goes to infinity. For the achievability scheme at hand, we obtain the optimal power allocation policy for the transmit signal and the AN in closed form. For the case of correlated fading channels, we show that the impact of the active eavesdropper can be completely removed if the transmit correlation matrices of the users and the eavesdropper are orthogonal. Inspired by this result, we propose a precoder null space design exploiting the low rank property of the transmit correlation matrices of massive MIMO channels, which can significantly degrade the eavesdropping capabilities of the active eavesdropper.
\end{abstract}

\section{INTRODUCTION}

Physical layer security has attracted significant research interest recently. In Wyner's pioneering work on informationtheoretic security, a "wiretap channel" model was defined and the associated secrecy capacity was obtained [1]. More recent studies have investigated the capacity and the precoder design for multiple antenna wiretap channels [2-4]. When the channel state information (CSI) of the eavesdropper is imperfectly known at the transmitter, artificial noise (AN) can be generated and transmitted along with the information-bearing signal to interfere the decoding process of the eavesdropper [5].

Most studies on physical layer security assume that perfect CSI of the legitimate channel is available at the transmitter and do not consider the channel training phase in acquiring the CSI. However, in time-division duplex (TDD) communication systems, the base station (BS) needs to estimate the channel for the subsequent downlink transmission based on the pilot sequences sent by the users in the uplink training phase. As a result, a smart eavesdropper might actively attack this channel training phase by sending the same pilot sequences as the users to cause pilot contamination at the transmitter. In this case, the eavesdropping capability can be improved significantly [6].

The emergence of smart mobile devices such as smart phones and wireless modems has led to an exponentially increasing demand for wireless data services. A recent and promising solution to meet this demand is massive multipleinput multiple-output (MIMO) technology, which utilizes a

Y. Wu, R. Schober, and D. W. K. Ng are with Institute for Digital Communications, Universität Erlangen-Nürnberg, Cauerstrasse 7, D-91058 Erlangen, Germany (Email: yongpeng.wu@lnt.de; schober@lnt.de; kwan@lnt.de).

C. Xiao is with the Department of Electrical and Computer Engineering, Missouri University of Science and Technology, Rolla, MO 65409, USA (Email: xiaoc@mst.edu).

G. Caire is with Institute for Telecommunication Systems, Technical University Berlin, Einsteinufer 25, 10587 Berlin, Germany (Email: caire@tuberlin.de). very large number of antennas at the BS with simple signal processing to serve a comparatively small (compared to the number of antennas) number of users. The field of massive MIMO communication systems was initiated by the pioneering work in [7] which considered multi-cell multi-user TDD communication. The key idea in [7] is that as the number of transmit antennas increases, due to the law of large numbers, the effects of uncorrelated receiver noise and fast fading vanish. Then, the only residual interference is caused by the reuse of the pilot sequences in other cells, which is known as pilot contamination. Since the publication of [7], many aspects of massive MIMO systems have been investigated [8-11].

Physical layer security for massive MIMO systems with passive eavesdroppers and imperfect CSI has been recently considered in [12]. Secure massive MIMO transmission for multi-cell multi-user systems has been investigated in [13], where a passive eavesdropper attempts to decode the information sent to one of the users. It was assumed in [12, 13] that the channel gains of both the desired receiver and the eavesdropper are independent and identically distributed (i.i.d.). The detection of the existence of an active eavesdropper in massive MIMO systems was investigated in [14] for i.i.d. fading channels. However, a systematic approach to combat the pilot contamination attack from the active eavesdropper and maintain secrecy of communication for general fading channels was not provided in [14] and has not been studied in the literature, yet.

In this paper, we study secure transmission for TDD multicell multi-user massive MIMO systems in the presence of an active eavesdropper over general fading channels. We assume that in the uplink training phase, the active eavesdropper sends the same pilot sequence as the desired receiver to impair the channel estimation at the transmitter. Therefore, pilot contamination occurs at the transmitter. Then, the transmitter uses the estimated channel to compute the precoder for downlink transmission.

This paper makes the following key contributions:

1) We derive a closed-form expression for the asymptotic achievable secrecy rate with matched filter precoding and AN generation at the transmitter assuming the number of transmit antennas tends to infinity.

2) Based on the derived asymptotic expression, we obtain the optimal power allocation policy for the information signal and the AN in closed form. Moreover, for the special case of point-to-point transmission over i.i.d. fading channels [6], we prove that the power allocated to the transmit signal should be less than a threshold in order to ensure reliable secure transmission.

3) For the case of correlated fading channels, we reveal that the impact of the active eavesdropper will vanish when the signal space (i.e., the span of the channel correlation matrix eigenvectors corresponding to nonzero eigenvalues) of the users and the eavesdropper are mutually orthogonal.

4) We exploit the low rank property of the transmit cor- 
relation matrices of massive MIMO channels $[9,10]$ to design an efficient precoding scheme that transmits in the null space of the transmit correlation matrix of the eavesdropper.

Notation: Vectors are denoted by lower-case bold-face letters; matrices are denoted by upper-case bold-face letters. Superscripts $(\cdot)^{T},(\cdot)^{*}$, and $(\cdot)^{H}$ stand for the matrix transpose, conjugate, and conjugate-transpose operations, respectively. We use $\operatorname{tr}(\mathbf{A})$ and $\mathbf{A}^{-1}$ to denote the trace operation and the inverse of matrix $\mathbf{A}$, respectively. $\|\cdot\|$ and $|\cdot|$ denote the Euclidean norm of a matrix/vector and a scalar, respectively. $\operatorname{diag}\{\mathbf{b}\}$ denotes a diagonal matrix. The main diagonal of $\operatorname{diag}\{\mathbf{b}\}$ contains the elements of vector $\mathbf{b}$. $\operatorname{vec}(\mathbf{A})$ stacks all columns of matrix A into a vector. The $M \times M$ identity matrix is denoted by $\mathbf{I}_{M}$, and the all-zero matrix is denoted by $\mathbf{0}$. The field of complex numbers is denoted by $\mathbb{C}$ and $E[\cdot]$ denotes statistical expectation. We use $\mathbf{x} \sim \mathcal{C N}\left(\mathbf{0}_{N}, \mathbf{R}\right)$ to denote a circularly symmetric complex Gaussian vector $\mathbf{x} \in \mathbb{C}^{N \times 1}$ with zero mean and covariance matrix $\mathbf{R}$. $[x]^{+}$ stands for $\max \{0, x\}, \otimes$ denotes the Kronecker product, and $A \stackrel{N \rightarrow \infty}{\rightarrow} B$ means that $A$ almost surely converges to $B$ when $N$ goes to infinity.

\section{SySTEM MODEL}

We consider a multi-cell multi-user system with $L+1$ cells. Each cell contains a BS with $N_{t}$ antennas and $K$ singleantenna users. Without loss of generality, let the reference cell to be denoted by $l=0$. An active eavesdropper with a singleantenna ${ }^{1}$ is located in the reference cell, and seeks to recover the private message destined to a specific target user $m$.

\section{A. Uplink Training and Channel Estimation}

In the uplink training and channel estimation phase, the received signal $\mathbf{y}_{0}$ at the $\mathrm{BS}$ in the reference cell is given by [9]

$$
\begin{aligned}
\mathbf{y}_{0}= & \sum_{k=1}^{K} \sqrt{P_{0 k}}\left(\boldsymbol{\omega}_{0 k} \otimes \mathbf{I}_{N_{t}}\right) \mathbf{h}_{0 k}^{0}+\sum_{l=1}^{L} \sum_{k=1}^{K} \sqrt{P_{l k}}\left(\boldsymbol{\omega}_{l k} \otimes \mathbf{I}_{N_{t}}\right) \mathbf{h}_{l k}^{0} \\
& +\sqrt{P_{E}}\left(\boldsymbol{\omega}_{0 m} \otimes \mathbf{I}_{N_{t}}\right) \mathbf{h}_{E}^{0}+\mathbf{n} .
\end{aligned}
$$

Here, $P_{l k}$ and $\boldsymbol{\omega}_{l k} \in \mathbb{C}^{\tau \times 1}$ are the average transmit power and the pilot sequence of the $k$ th user in the $l$ th cell, where $\tau$ denotes the length of the pilot sequence. $\mathbf{h}_{l k}^{p}=\left(\mathbf{R}_{l k}^{p}\right)^{1 / 2} \mathbf{g}_{l k}^{p} \in$ $\mathbb{C}^{N_{t} \times 1}$ denotes the channel between the $k$ th user in the $l$ th cell and the BS in the $p$ th cell, where $\mathbf{g}_{l k}^{p} \sim \mathcal{C} \mathcal{N}\left(\mathbf{0}_{N_{t}}, \mathbf{I}_{N_{t}}\right)$ and $\mathbf{R}_{l k}^{p} \in \mathbb{C}^{N_{t} \times N_{t}}$ is the correlation matrix of channel $\mathbf{h}_{l k}^{p}$. $P_{E}$ denotes the average transmit power of the eavesdropper. $\mathbf{h}_{E}^{l}=\left(\mathbf{R}_{E}^{l}\right)^{1 / 2} \mathbf{g}_{E}^{l}$ denotes the channel between the eavesdropper and the BS in the $l$ th cell, where $\mathbf{g}_{E}^{l} \sim \mathcal{C N}\left(\mathbf{0}_{N_{t}}, \mathbf{I}_{N_{t}}\right)$ and $\mathbf{R}_{E}^{l} \in \mathbb{C}^{N_{t} \times N_{t}}$ is the correlation matrix of channel $\mathbf{h}_{E}^{l} . \mathbf{n} \in \mathbb{C}^{\tau N_{t} \times 1}$ is a zero-mean complex Gaussian noise vector with covariance matrix $N_{0} \mathbf{I}_{\tau N_{t}}$. We assume that the same $K$ orthogonal pilot sequences are used for the $K$ users in each cell [7], i.e., $\boldsymbol{\omega}_{0 k}=\boldsymbol{\omega}_{1 k}=\cdots \boldsymbol{\omega}_{L k}=\boldsymbol{\omega}_{k}$, $\boldsymbol{\omega}_{l k}^{H} \boldsymbol{\omega}_{l k}=\tau, \boldsymbol{\omega}_{l k}^{H} \boldsymbol{\omega}_{l p}=0$. Then, the minimum mean square

\footnotetext{
${ }^{1}$ In order to obtain some initial insights regarding massive MIMO systems with active eavesdropping, we assume a single-antenna eavesdropper in this paper. It is noted that even a single-antenna active eavesdropper is an extremely harmful threat to secrecy transmission since it influences the precoder used in downlink transmission [6,14]. Considering the conclusion in [15, Appendix B], we can also extend our analysis to multiple-antenna eavesdroppers. However, the extension to multiple-antenna eavesdroppers requires additional pilot sequence design at the eavesdropper and a modified precoder design at the transmitter side, which will be investigated in the journal version of this paper.
}

error (MMSE) estimate of $\mathbf{h}_{0 m}^{0}$ is given by [16]

$$
\begin{aligned}
& \widehat{\mathbf{h}}_{0 m}^{0}= \\
& \sqrt{P_{0 m}} \mathbf{R}_{0 m}^{0}\left(N_{0} \mathbf{I}_{N_{t}}+\tau\left(\sum_{t=0}^{L} P_{t m} \mathbf{R}_{t m}^{0}+P_{E} \mathbf{R}_{E}^{0}\right)\right)^{-1} \widetilde{\mathbf{y}}_{0 m},
\end{aligned}
$$

where
$\widetilde{\mathbf{y}}_{0 m}=\sqrt{P_{0 m}} \tau \mathbf{h}_{0 m}^{0}+\sum_{t=1}^{L} \sqrt{P_{t m}} \tau \mathbf{h}_{t m}^{0}+\sqrt{P_{E}} \tau \mathbf{h}_{E}^{0}+\left(\boldsymbol{\omega}_{m} \otimes \mathbf{I}_{N_{t}}\right)^{H} \mathbf{n}$.

Here, $\mathbf{h}_{0 m}^{0}=\widehat{\mathbf{h}}_{0 m}^{0}+\mathbf{e}_{0 m}^{0}$, where the estimated channel $\widehat{\mathbf{h}}_{0 m}^{0} \sim \mathcal{C N}\left(\mathbf{0}_{N_{t}}, \widehat{\mathbf{R}}_{0 m}^{0}\right)$ and the estimation error $\mathbf{e}_{0 m}^{0} \sim$ $\mathcal{C N}\left(\mathbf{0}_{N_{t}}, \mathbf{R}_{0 m}^{0}-\widehat{\mathbf{R}}_{0 m}^{0}\right)$ are mutually independent. The correlation matrix $\widehat{\mathbf{R}}_{0 m}^{0}$ is given by

$$
\begin{aligned}
& \widehat{\mathbf{R}}_{0 m}^{0}= \\
& P_{0 m} \tau \mathbf{R}_{0 m}^{0}\left(N_{0} \mathbf{I}_{N_{t}}+\tau\left(\sum_{t=0}^{L} P_{t m} \mathbf{R}_{t m}^{0}+P_{E} \mathbf{R}_{E}^{0}\right)\right)^{-1} \mathbf{R}_{0 m}^{0} .
\end{aligned}
$$

Similarly, the MMSE channel estimates for the $m$ th user and the $k$ th user, $k=1,2, \cdots, K, k \neq m$, in the lth cell, $l=0,1, \cdots, L$, are given by

$$
\begin{aligned}
& \widehat{\mathbf{h}}_{l m}^{l}= \\
& \sqrt{P_{l m}} \mathbf{R}_{l m}^{l}\left(N_{0} \mathbf{I}_{N_{t}}+\tau\left(\sum_{t=0}^{L} P_{t k} \mathbf{R}_{t m}^{l}+P_{E} \mathbf{R}_{E}^{l}\right)\right)^{-1} \widetilde{\mathbf{y}}_{l m} \\
& \widetilde{\mathbf{y}}_{l m}=\sqrt{P_{l m}} \tau \mathbf{h}_{l m}^{l}+\sum_{t=0, t \neq l}^{L} \sqrt{P_{t m}} \tau \mathbf{h}_{t m}^{l} \\
& +\sqrt{P_{E}} \tau \mathbf{h}_{E}^{l}+\left(\boldsymbol{\omega}_{m} \otimes \mathbf{I}_{N_{t}}\right)^{H} \mathbf{n} \\
& \widehat{\mathbf{h}}_{l k}^{l}=\sqrt{P_{l k}} \mathbf{R}_{l k}^{l}\left(N_{0} \mathbf{I}_{N_{t}}+\tau \sum_{t=0}^{L} P_{t k} \mathbf{R}_{t k}^{l}\right)^{-1} \widetilde{\mathbf{y}}_{l k} \\
& \widetilde{\mathbf{y}}_{l k}=\sqrt{P_{l k}} \tau \mathbf{h}_{l k}^{l}+\sum_{t=0, t \neq l}^{L} \sqrt{P_{t m}} \tau \mathbf{h}_{t k}^{l}+\left(\boldsymbol{\omega}_{k} \otimes \mathbf{I}_{N_{t}}\right)^{H} \mathbf{n},
\end{aligned}
$$

respectively. The correlation matrices of $\widehat{\mathbf{h}}_{l m}^{l}$ and $\widehat{\mathbf{h}}_{l k}^{l}$ are given by

$$
\widehat{\mathbf{R}}_{l m}^{l}=P_{l m} \tau \mathbf{R}_{l m}^{l}\left(N_{0} \mathbf{I}_{N_{t}}+\tau\left(\sum_{t=0}^{L} P_{t k} \mathbf{R}_{t m}^{l}+P_{E} \mathbf{R}_{E}^{l}\right)\right)^{-1} \mathbf{R}_{l m}^{l}
$$

and

$$
\widehat{\mathbf{R}}_{l k}^{l}=P_{l k} \tau \mathbf{R}_{l k}^{l}\left(N_{0} \mathbf{I}_{N_{t}}+\tau \sum_{t=0}^{L} P_{t k} \mathbf{R}_{t k}^{l}\right)^{-1} \mathbf{R}_{l k}^{l},
$$

respectively.

Remark 1: We assume that the correlation matrices of the users and the eavesdropper are perfectly known at the transmitter, see (2), (5), (7). For massive MIMO systems, it is reasonable to assume that the statistical CSI of the users of the system is known at the BS [9]. Therefore, if the BS attempts to transmit a private message to some users and treats other users as eavesdroppers, i.e., the eavesdropper is an idle user of the system, the correlation matrix of the eavesdropper can also assumed to be known. In other cases, the assumption that the correlation matrices of the active eavesdropper are available at the transmitter may also be reasonable. For example, we can obtain $E\left[\widetilde{\mathbf{y}}_{l m} \widetilde{\mathbf{y}}_{l m}^{H}\right]$ by averaging $\widetilde{\mathbf{y}}_{l m}$ over different data slots. Eq. (6) suggests that $E\left[\widetilde{\mathbf{y}}_{l m} \widetilde{\mathbf{y}}_{l m}^{H}\right]$ is the sum of the correlation matrices of all users and the eavesdropper. Then, $P_{E} \mathbf{R}_{E}^{l}$ can be obtained by subtracting the correlation matrices of the legitimate users and the noise from $E\left[\widetilde{\mathbf{y}}_{l m} \widetilde{\mathbf{y}}_{l m}^{H}\right]$. 


\section{B. Downlink Data Transmission}

Next, we consider data transmission. We assume that the BSs in all $L+1$ cells perform jamming to prevent eavesdropping in their own cell. Then, the transmit signal in the $l$ th cell $l=0,1, \cdots, L$ is given by

$$
\mathbf{x}_{l}=\sqrt{P}\left(\sqrt{p} \sum_{k=1}^{K} \mathbf{w}_{l k} s_{l k}+\sqrt{q} \mathbf{U}_{\mathrm{null}, l} \mathbf{z}_{l}\right),
$$

where $P$ is the average transmit power for downlink transmission and $s_{l k}$ is the transmit signal for the $k$ th user in the $l$ th cell with $E\left[\left|s_{l k}\right|^{2}\right]=1 . p$ and $q$ represent the power allocation between the transmit signal and the AN with $p+q=1$. Due to the high implementation complexity of the matrix inversion required for zero forcing and MMSE precoding, here we adopt the simple matched filter precoding for massive MIMO systems [7, 8, 13]. Thus, we set $\mathbf{w}_{l k}=\frac{\widehat{\mathbf{h}}_{l k}^{l}}{\left\|\widehat{\mathbf{h}}_{l k}^{l}\right\|}$ for the $k$ th user in the $l$ th cell. $\mathbf{U}_{\text {null, } l}$ and $\mathbf{z}_{l} \sim \mathcal{C N}\left(\mathbf{0}_{N_{t}}, \mathbf{I}_{N_{t}}\right)$ denote the AN shaping matrix and the AN vector in the $l$ th cell, respectively. We define $\widehat{\mathbf{H}}_{l}^{l}=\left[\widehat{\mathbf{h}}_{l 1}^{l}, \widehat{\mathbf{h}}_{l 2}^{l}, \cdots, \widehat{\mathbf{h}}_{l K}^{l}\right]$. To reduce the implementation complexity when the number of antennas is large, we fix the AN shaping matrix to be the asymptotic null space of $\widehat{\mathbf{H}}_{l}^{l}$. Based on [17, Corollary 1], we have $\frac{1}{N_{t}}\left(\widehat{\mathbf{H}}_{l}^{l}\right)^{H} \widehat{\mathbf{H}}_{l}^{l} \stackrel{N_{t} \rightarrow \infty}{\rightarrow} \frac{1}{N_{t}} \operatorname{diag}\left[\operatorname{tr}\left(\widehat{\mathbf{R}}_{l 1}^{l}\right), \operatorname{tr}\left(\widehat{\mathbf{R}}_{l 2}^{l}\right), \cdots, \operatorname{tr}\left(\widehat{\mathbf{R}}_{l K}^{l}\right)\right]$. Thus, we set $\mathbf{U}_{\text {null, } l}=\mathbf{I}_{N_{t}}$ $\widehat{\mathbf{H}}_{l}^{l} \operatorname{diag}\left[\operatorname{tr}\left(\widehat{\mathbf{R}}_{l 1}^{l}\right)^{-1}, \operatorname{tr}\left(\widehat{\mathbf{R}}_{l 2}^{l}\right)^{-1}, \cdots, \operatorname{tr}\left(\widehat{\mathbf{R}}_{l K}^{l}\right)^{-1}\right]\left(\widehat{\mathbf{H}}_{l}^{l}\right)^{H}$. It can be shown that $\frac{1}{N_{t}} \operatorname{tr}\left(\mathbf{U}_{\text {null, }, l} \mathbf{U}_{\text {null, }, l}^{H}\right) \stackrel{N_{t} \rightarrow \infty}{\rightarrow} \frac{1}{N_{t}}\left(N_{t}-K\right)$. To ensure $\frac{1}{N_{t}} \mathbf{x}_{l}^{H} \mathbf{x}_{l} \stackrel{N_{t} \rightarrow \infty}{\rightarrow} \frac{1}{N_{t}} P$, we set $K p+\left(N_{t}-K\right) q=1$.

The received signal at the $m$ th user in the reference cell, $y_{0 m}$, and at the eavesdropper, $y_{\text {eve }}$, are given by

$$
y_{0 m}=\sum_{l=0}^{L}\left(\mathbf{h}_{0 m}^{l}\right)^{H} \mathbf{x}_{l}+n_{0 m}
$$

and

$$
y_{\text {eve }}=\sum_{l=0}^{L}\left(\mathbf{h}_{E}^{l}\right)^{H} \mathbf{x}_{l}+n_{\mathrm{eve}}
$$

respectively. Here, $n_{0 m}$ and $n_{\text {eve }}$ are zero-mean Gaussian noise processes with variance $N_{0, \mathrm{~d}}$. We define the signal-to-noise ratio (SNR) of the downlink transmission as $\gamma=P / N_{0, \mathrm{~d}}$.

\section{Matched Filter Precoding And AN Generation}

An achievable ergodic secrecy rate of massive MIMO systems in Section II can be expressed as [13]

$$
R_{\mathrm{sec}}=\left[R_{0 m}-C_{\mathrm{eve}}\right]^{+}
$$

where $R_{0 m}$ and $C_{\text {eve }}$ denote the achievable ergodic rate between the BS and the $m$ th user and the ergodic capacity between the BS and the eavesdropper, in the 0th cell, respectively. The achievable ergodic rate $R_{0 m}$ is given by $[13, \mathrm{Eq}$. (8)]

$$
R_{0 m}=E\left[\log _{2}\left(1+\operatorname{SINR}_{0 m}\right)\right] .
$$

where $\mathrm{SINR}_{0 m}$ is given by

$$
\mathrm{SINR}_{0 m}=\frac{p \gamma\left|\left(\mathbf{h}_{0 m}^{0}\right)^{H} \mathbf{w}_{0 m}\right|^{2}}{A}
$$

$$
\begin{aligned}
& A=p \gamma \sum_{k=1, k \neq m}^{K}\left[\left|\left(\mathbf{h}_{0 m}^{0}\right)^{H} \mathbf{w}_{0 k}\right|^{2}\right]+q \gamma\left[\left|\left(\mathbf{h}_{0 m}^{0}\right)^{H} \mathbf{U}_{\mathrm{null}, 0}\right|^{2}\right]+p \gamma \\
& \times \sum_{l=1}^{L}\left[\sum_{k=1}^{K}\left[\left|\left(\mathbf{h}_{0 m}^{l}\right)^{H} \mathbf{w}_{l k}\right|^{2}\right]+q \gamma \sum_{l=1}^{L}\left[\left|\left(\mathbf{h}_{0 m}^{l}\right)^{H} \mathbf{U}_{\mathrm{null}, l}\right|^{2}\right]\right]+1 .
\end{aligned}
$$

To capture the worst case, we assume that the eavesdropper has perfect knowledge of its own channel and is able to decode and cancel the signals of all intra-cell and inter-cell users from the received signal $\mathbf{y}_{\text {eve }}$ in (13) except for the signal intended for the $m$ th user in the 0th cell. In this case, $C_{\text {eve }}$ can be expressed as [13, Eq. (7)]

$$
\begin{aligned}
& \text { where } \\
& B=q \gamma\left(\mathbf{h}_{E}^{0}\right){ }^{H} \mathbf{U}_{\text {null, }, 0} \mathbf{U}_{\text {null, }, 0}^{H} \mathbf{h}_{E}^{0}+q \gamma \sum_{l=1}^{L}\left(\mathbf{h}_{E}^{l}\right){ }^{H} \mathbf{U}_{\text {null, }, l} \mathbf{U}_{\text {null, },}^{H} \mathbf{h}_{E}^{l}+1 .
\end{aligned}
$$

Now, we are ready to provide an asymptotic achievable secrecy rate expression when the number of antennas tends to infinity, which is given in the following proposition.

Proposition 1: An asymptotic achievable secrecy rate for matched filter precoding and AN generation for multi-cell multi-user massive MIMO systems with an active eavesdropper is given by

$$
\begin{aligned}
R_{\text {sec, asy }} & \stackrel{N_{t} \rightarrow \infty}{\rightarrow}\left[\log _{2}\left(1+\mathrm{SINR}_{0 m, \text { asy }}\right)\right. \\
& \left.-\log _{2}\left(1+\mathrm{SINR}_{\text {eve }, \text { asy }}\right)\right]^{+},
\end{aligned}
$$

where

$$
\begin{aligned}
\mathrm{SINR}_{0 m, \text { asy }} & =\frac{p \gamma \theta_{m}}{p \gamma \theta_{b, p}+q \gamma \theta_{b, q}+1} \\
\mathrm{SINR}_{\text {eve, asy }} & =\frac{p \gamma \theta_{e, e}}{q \gamma \theta_{e, q}+1}
\end{aligned}
$$

with

$$
\begin{aligned}
\theta_{m}= & \operatorname{tr}\left(\widehat{\mathbf{R}}_{0 m}^{0}\right)+\operatorname{tr}\left(\widehat{\mathbf{R}}_{0 m}^{0}\right)^{-1} \operatorname{tr}\left(\left(\mathbf{R}_{0 m}^{0}-\widehat{\mathbf{R}}_{0 m}^{0}\right) \widehat{\mathbf{R}}_{0 m}^{0}\right) \\
\theta_{b, p}= & \sum_{l=0}^{L} \sum_{k=1, k \neq m}^{K} \operatorname{tr}\left(\widehat{\mathbf{R}}_{l k}^{l}\right)^{-1} \operatorname{tr}\left(\mathbf{R}_{0 m}^{l} \widehat{\mathbf{R}}_{l k}^{l}\right)+\sum_{l=1}^{L} \operatorname{tr}\left(\widehat{\mathbf{R}}_{l m}^{l}\right)^{-1} \Lambda_{0 m}^{l} \\
\theta_{b, q}= & \sum_{l=0}^{L} \operatorname{tr}\left(\mathbf{R}_{0 m}^{l}\right)-\sum_{l=0}^{L} \sum_{k=1, k \neq m}^{K} \operatorname{tr}\left(\widehat{\mathbf{R}}_{l k}^{l}\right)^{-1} \operatorname{tr}\left(\mathbf{R}_{0 m}^{l} \widehat{\mathbf{R}}_{l k}^{l}\right) \\
& -\sum_{l=0}^{L} \operatorname{tr}\left(\widehat{\mathbf{R}}_{l m}^{l}\right)^{-1} \Lambda_{0 m}^{l} \\
\Lambda_{0 m}^{l} & =\tau^{2} P_{0 m}\left|\operatorname{tr}\left(\mathbf{C}_{l m}^{l} \mathbf{R}_{0 m}^{l}\right)\right|^{2}+\tau N_{0} \operatorname{tr}\left(\mathbf{R}_{0 m}^{l} \mathbf{C}_{l m}^{l}\left(\mathbf{C}_{l m}^{l}\right)^{H}\right) \\
& +\tau^{2} \sum_{t=1}^{L} P_{t m} \operatorname{tr}\left(\mathbf{R}_{0 m}^{l} \mathbf{C}_{l m}^{l} \mathbf{R}_{t m}^{l}\left(\mathbf{C}_{l m}^{l}\right)^{H}\right) \\
& +\tau^{2} P_{E} \operatorname{tr}\left(\mathbf{R}_{0 m}^{l} \mathbf{C}_{l m}^{l} \mathbf{R}_{E}^{l}\left(\mathbf{C}_{l m}^{l}\right)^{H}\right) \\
\mathbf{C}_{l m}^{l} & =\sqrt{P_{l m}} \mathbf{R}_{l m}^{l}\left(N_{0} \mathbf{I}_{N_{t}}+\tau\left(\sum_{t=0}^{L} P_{t m} \mathbf{R}_{t m}^{l}+P_{E} \mathbf{R}_{E}^{l}\right)\right)^{-1}
\end{aligned}
$$

$$
\theta_{e, e}=\frac{\Lambda_{E}^{0}}{\operatorname{tr}\left(\widehat{\mathbf{R}}_{0 m}^{0}\right)}
$$




$$
\begin{aligned}
\theta_{e, q} & =\sum_{l=0}^{L} \operatorname{tr}\left(\mathbf{R}_{E}^{l}\right)-\sum_{l=0}^{L} \sum_{k=1, k \neq m}^{K} \operatorname{tr}\left(\widehat{\mathbf{R}}_{l k}^{l}\right)^{-1} \operatorname{tr}\left(\mathbf{R}_{E}^{l} \widehat{\mathbf{R}}_{l k}^{l}\right) \\
& -\sum_{l=0}^{L} \operatorname{tr}\left(\widehat{\mathbf{R}}_{l m}^{l}\right)^{-1} \Lambda_{E}^{l} \\
\Lambda_{E}^{l} & =\tau^{2} P_{E}\left|\operatorname{tr}\left(\mathbf{C}_{l m}^{l} \mathbf{R}_{E}^{l}\right)\right|^{2}+\tau N_{0} \operatorname{tr}\left(\mathbf{R}_{E}^{l} \mathbf{C}_{l m}^{l}\left(\mathbf{C}_{l m}^{l}\right)^{H}\right) \\
& +\tau^{2} \sum_{t=0}^{L} P_{t m} \operatorname{tr}\left(\mathbf{R}_{E}^{l} \mathbf{C}_{l m}^{l} \mathbf{R}_{t m}^{l}\left(\mathbf{C}_{l m}^{l}\right)^{H}\right) .
\end{aligned}
$$

Proof: See Appendix A.

Based on Proposition 1, we can obtain several new insights for massive MIMO systems with an active eavesdropper, which are summarized in the following corollaries. The proofs of these corollaries can be directly obtained by finding feasible $p$ which maximize $R_{\text {sec, asy }}$ or yield $R_{\text {sec, asy }}>0$ in (21).

Corollary 1: Let us define

$p_{1}=$

$$
\frac{-\left(a_{1} c_{2}-a_{2} c_{1}\right)-\sqrt{\left(a_{1} c_{2}-a_{2} c_{1}\right)^{2}-\left(a_{1} b_{2}-a_{2} b_{1}\right)\left(b_{1}-b_{2}\right) c_{1}}}{\left(a_{1} b_{2}-a_{2} b_{1}\right)}
$$

and

$$
\begin{aligned}
& p_{2}= \\
& \frac{-\left(a_{1} c_{2}-a_{2} c_{1}\right)+\sqrt{\left(a_{1} c_{2}-a_{2} c_{1}\right)^{2}-\left(a_{1} b_{2}-a_{2} b_{1}\right)\left(b_{1}-b_{2}\right) c_{1}}}{\left(a_{1} b_{2}-a_{2} b_{1}\right)}
\end{aligned}
$$

where

$$
\begin{aligned}
a_{1} & =-\gamma^{2}\left(\left(N_{t}-K\right) \theta_{m}-\left(N_{t}-K\right) \theta_{b, p}-K \theta_{b, q}\right) K \theta_{e, q} \\
b_{1} & =\gamma\left(\left(N_{t}-K\right) \theta_{m}+\left(N_{t}-K\right) \theta_{b, p}-K \theta_{b, q}\right) \\
& \times\left(\gamma \theta_{e, q}+N_{t}-K\right)+\gamma\left(\gamma \theta_{b, q}+N_{t}-K\right) \\
& \times\left(\left(N_{t}-K\right) \theta_{e, q}-K \theta_{e, q}\right) \\
c_{1} & =\left(\gamma \theta_{b, q}+N_{t}-K\right)\left(\gamma \theta_{e, q}+N_{t}-K\right) \\
a_{2} & =\gamma^{2}\left(\left(N_{t}-K\right) \theta_{b, p}-K \theta_{b, q}\right)\left(\left(N_{t}-K\right) \theta_{e, e}-K \theta_{e, q}\right) \\
b_{2} & =\gamma\left(\gamma \theta_{b, q}+N_{t}-K\right)\left(\left(N_{t}-K\right) \theta_{e, e}-K \theta_{e, q}\right) \\
& +\gamma\left(\left(N_{t}-K\right) \theta_{b, p}-K \theta_{b, q}\right)\left(\gamma \theta_{e, q}+N_{t}-K\right) \\
c_{2} & =\left(\gamma \theta_{b, q}+N_{t}-K\right)\left(\gamma \theta_{e, q}+N_{t}-K\right)
\end{aligned}
$$

Then, the optimal power allocation $p$ maximizing the asymptotic achievable secrecy rate in (21) is given by

$$
p^{*}=\left\{\begin{array}{lll}
1, & \text { if } & p_{1} \notin[0,1], p_{2} \notin[0,1], \\
\arg \max _{p \in\left\{1, p_{1}\right\}} R_{\text {sec, asy }}(p), & \text { if } & p_{1} \in[0,1], p_{2} \notin[0,1], \\
\arg \max _{p \in\left\{1, p_{2}\right\}} R_{\text {sec, asy }}(p), & \text { if } & p_{1} \notin[0,1], p_{2} \in[0,1], \\
\arg \max _{p \in\left\{1, p_{1}, p_{2}\right\}} R_{\text {sec, asy }}(p), & \text { if } & p_{1} \in[0,1], p_{2} \in[0,1] .
\end{array}\right.
$$

Corollary 2: For $R_{\text {sec, asy }}>0$, the power allocated to the transmit signal must satisfy ${ }^{2}$ :

$$
\begin{aligned}
& p>-\frac{\left(b_{1}-b_{2}\right)}{\left(a_{1}-a_{2}\right)} \text {, if } \quad\left(a_{1}-a_{2}\right)>0 ; \\
& p<-\frac{\left(b_{1}-b_{2}\right)}{\left(a_{1}-a_{2}\right)} \text {, if }\left(a_{1}-a_{2}\right)<0 .
\end{aligned}
$$

For the special case of single-cell single-user communication $(L=0, K=1, m=1)$ with i.i.d. fading $\left(\mathbf{R}_{01}^{0}=\beta_{B} \mathbf{I}_{N_{t}}\right.$ and $\mathbf{R}_{E}^{0}=\beta_{E} \mathbf{I}_{N_{t}}$, where $\beta_{B}$ and $\beta_{E}$ denote the path-loss for the desired user and the eavesdropper, respectively. ) [6], we have the following result.

Corollary 3: $p<p_{\mathrm{th}, 1}$ has to hold in order to achieve secure transmission in single-cell single-user communication

\footnotetext{
${ }^{2}$ When $a_{1}-a_{2}=0$, if $b_{1}-b_{2}>0$, then secure transmission can be achieved for any $p$; otherwise, secure transmission can not be achieved regardless of the value of $p$.
}

with i.i.d. fading, where

$$
\begin{array}{r}
p_{\mathrm{th}, 1}=1+\frac{\left(N_{0}+\tau\left(P_{01} \beta_{B}+P_{E} \beta_{E}\right)\right)\left(P_{01} \beta_{B}^{2}-P_{E} \beta_{E}^{2}\right)}{P_{01} \beta_{B}^{2} \gamma \beta_{E}\left(N_{0}+\tau P_{01} \beta_{B}\right)} \\
-\frac{P_{E} \beta_{E}\left(N_{0}+\tau P_{E} \beta_{E}\right)}{P_{01} \beta_{B}\left(N_{0}+\tau P_{01} \beta_{B}\right)} .
\end{array}
$$

$p_{\mathrm{th}, 1}$ is a decreasing function of $\gamma$. In the high SNR regime, when $\gamma \rightarrow \infty$,

$$
p_{\mathrm{th}, 1} \stackrel{\gamma \rightarrow \infty}{\rightarrow} 1-\frac{P_{E} \beta_{E}\left(N_{0}+\tau P_{E} \beta_{E}\right)}{P_{01} \beta_{B}\left(N_{0}+\tau P_{01} \beta_{B}\right)} .
$$

Remark 2: We note from (41) that for single-cell single-user communication with i.i.d. fading, to enable secure transmission, the power allocated to the transmit signal has to decrease with increasing SNR. In the high SNR regime, as shown in (42), if the eavesdropper increases the average transmit power $P_{E}$ in the uplink training phase to make $p_{\mathrm{th}, 1}<0$, secure transmission cannot be achieved due to the pilot contamination attack. In this case, increasing the average transmit power of the desired user $P_{01}$ in the uplink training phase is essential to ensure secure transmission.

\section{Null SpaCe Transmission Design}

In the following proposition, we investigate the asymptotic achievable secrecy rate under a special condition where the transmit correlation matrices of the users are orthogonal to the transmit correlation matrices of the eavesdropper.

Proposition 2: If $\sum_{t=0}^{L} P_{t m} \operatorname{tr}\left(\mathbf{R}_{t m}^{l} \mathbf{R}_{E}^{l}\right)=0$ for $l=$ $0,1, \cdots, L$, then the secrecy rate $R_{\mathrm{sec} \text {, asy }}$ is equivalent to the rate without the eavesdropper $R_{\text {sec, asy, orth }}=\log _{2}(1+$ SINR $_{0 m, \text { asy, orth }}$, where

$$
\begin{aligned}
& \mathrm{SINR}_{0 m, \text { asy }, \text { orth }}=\frac{p \gamma \theta_{m, \text { orth }}}{p \gamma \theta_{b, p, \text { orth }}+q \gamma \theta_{b, q, \text { orth }}+1} \\
& { }_{\theta_{m, \text { orth }}}^{\text {with }}=\operatorname{tr}\left(\widehat{\mathbf{R}}_{0 m, \text { orth }}^{0}\right) \\
& +\operatorname{tr}\left(\widehat{\mathbf{R}}_{0 m, \text { orth }}^{0}\right)^{-1} \operatorname{tr}\left(\left(\mathbf{R}_{0 m}^{0}-\widehat{\mathbf{R}}_{0 m, \text { orth }}^{0}\right) \widehat{\mathbf{R}}_{0 m, \text { orth }}^{0}\right) \\
& \theta_{b, p, \text { orth }}=\sum_{l=0}^{L} \sum_{k=1, k \neq m}^{K} \operatorname{tr}\left(\widehat{\mathbf{R}}_{l k}^{l}\right)^{-1} \operatorname{tr}\left(\mathbf{R}_{0 m}^{l} \widehat{\mathbf{R}}_{l k}^{l}\right) \\
& +\sum_{l=1}^{L} \operatorname{tr}\left(\widehat{\mathbf{R}}_{l m, \text { orth }}^{l}\right)^{-1} \Lambda_{0 m, \text { orth }}^{l} \\
& \theta_{b, q, \text { orth }}=\sum_{l=0}^{L} \operatorname{tr}\left(\mathbf{R}_{0 m}^{l}\right)-\sum_{l=0}^{L} \sum_{k=1, k \neq m}^{K} \operatorname{tr}\left(\widehat{\mathbf{R}}_{l k}^{l}\right)^{-1} \operatorname{tr}\left(\mathbf{R}_{0 m}^{l} \widehat{\mathbf{R}}_{l k}^{l}\right) \\
& -\sum_{l=0}^{L} \operatorname{tr}\left(\widehat{\mathbf{R}}_{l m, \text { orth }}^{l}\right)^{-1} \Lambda_{0 m, \text { orth }}^{l} \\
& \Lambda_{0 m, \text { orth }}^{l}=\tau^{2} P_{0 m}\left|\operatorname{tr}\left(\mathbf{C}_{l m, \text { orth }}^{l} \mathbf{R}_{0 m}^{l}\right)\right|^{2} \\
& +\tau^{2} \sum_{t=1}^{L} P_{t m} \operatorname{tr}\left(\mathbf{R}_{0 m}^{l} \mathbf{C}_{l m, \text { orth }}^{l} \mathbf{R}_{t m}^{l}\left(\mathbf{C}_{l m, \text { orth }}^{l}\right)^{H}\right) \\
& +\tau N_{0} \operatorname{tr}\left(\mathbf{R}_{0 m}^{l} \mathbf{C}_{l m, \text { orth }}^{l}\left(\mathbf{C}_{l m, \text { orth }}^{l}\right)^{H}\right) \\
& \mathbf{C}_{l m, \text { orth }}^{l}=\sqrt{P_{l m}} \mathbf{R}_{l m}^{l}\left(N_{0} \mathbf{I}_{N_{t}}+\tau \sum_{t=0}^{L} P_{t m} \mathbf{R}_{t m}^{l}\right)^{-1} \\
& \widehat{\mathbf{R}}_{l m, \text { orth }}^{l}=P_{l m} \tau \mathbf{R}_{l m}^{l}\left(N_{0} \mathbf{I}_{N_{t}}+\tau \sum_{t=0}^{L} P_{t m} \mathbf{R}_{t m}^{l}\right)^{-1} \mathbf{R}_{l m}^{l} .
\end{aligned}
$$

Proof: The proposition can be proved by applying the matrix inversion lemma in (4) and (9), using the fact $\mathbf{R}_{E}^{0} \mathbf{C}_{0 m}^{0}=$ $\mathbf{0}$, and performing some matrix transformation operations. Due to space limitation, details of the proof are omitted here. 
Proposition 2 shows that when the channels of the eavesdropper and the users are statistically orthogonal, the impact of the pilot contamination attack disappears. It is known that for many massive MIMO scenarios, the transmit correlation matrices of the channels are low rank $[9,10]$. As a result, inspired by Proposition 2, we propose a null space transmission design along the correlation matrix of the eavesdropper's channel $\mathbf{h}_{E}^{0}$ as follows.

In the uplink training phase, we can rewrite (1) as

$\mathbf{Y}_{0}=\sum_{k=1}^{K} \sqrt{P_{0 k}} \mathbf{h}_{0 k}^{0} \boldsymbol{\omega}_{k}^{T}+\sum_{l=1}^{L} \sum_{k=1}^{K} \sqrt{P_{l k}} \mathbf{h}_{l k}^{0} \boldsymbol{\omega}_{k}^{T}+\sqrt{P_{E}} \mathbf{h}_{E}^{0} \boldsymbol{\omega}_{m}^{T}+\mathbf{N}$

where $\mathbf{y}_{0}=\operatorname{vec}\left(\mathbf{Y}_{0}\right)$ and $\mathbf{n}=\operatorname{vec}(\mathbf{N})$. Assume the rank of $\mathbf{R}_{E}^{0}$ is $N$. Let us now construct a matrix $\mathbf{V}_{E}^{0} \in \mathbb{C}^{N_{t} \times M}$, whose $M$ columns are the $M$ eigenvectors which correspond to the zero eigenvalues of $\mathbf{R}_{E}^{0}$, where $M=N_{t}-N$. In the uplink training phase, we multiply $\mathbf{Y}_{0}$ with $\mathbf{V}_{E}^{0}$ to obtain

$$
\begin{aligned}
& \left(\mathbf{V}_{E}^{0}\right)^{H} \mathbf{Y}_{0}=\sum_{k=1}^{K} \sqrt{P_{0 k}}\left(\mathbf{V}_{E}^{0}\right)^{H} \mathbf{h}_{0 k}^{0} \boldsymbol{\omega}_{k}^{T}+\sum_{l=1}^{L} \sum_{k=1}^{K} \sqrt{P_{l k}} \\
& \times\left(\mathbf{V}_{E}^{0}\right)^{H} \mathbf{h}_{l k}^{0} \boldsymbol{\omega}_{k}^{T}+\sqrt{P_{E}}\left(\mathbf{V}_{E}^{0}\right)^{H} \mathbf{h}_{E}^{0} \boldsymbol{\omega}_{m}^{T}+\left(\mathbf{V}_{E}^{0}\right)^{H} \mathbf{N} \\
& \mathbf{y}_{0, \text { null }}=\operatorname{vec}\left(\left(\mathbf{V}_{E}^{0}\right)^{H} \mathbf{Y}_{0}\right)=\sum_{k=1}^{K} \sqrt{P_{0 k}}\left(\boldsymbol{\omega}_{k} \otimes \mathbf{I}_{N_{t}}\right) \mathbf{h}_{0 k, \text { null }}^{0} \\
& +\sum_{l=1}^{L} \sum_{k=1}^{K} \sqrt{P_{l k}}\left(\boldsymbol{\omega}_{k} \otimes \mathbf{I}_{N_{t}}\right) \mathbf{h}_{l k, \text { null }}^{0} \\
& +\sqrt{P_{E}}\left(\boldsymbol{\omega}_{m} \otimes \mathbf{I}_{N_{t}}\right)\left(\mathbf{V}_{E}^{0}\right)^{H} \mathbf{h}_{E}^{0}+\widetilde{\mathbf{n}}_{\text {null }}
\end{aligned}
$$

where $\mathbf{h}_{l k, \text { null }}^{0}=\left(\mathbf{V}_{E}^{0}\right)^{H} \mathbf{h}_{l k}^{0} \sim \mathcal{C N}\left(0, \mathbf{R}_{l k, \text { null }}^{0}\right), \mathbf{R}_{l k, \text { null }}^{0}=$ $\left(\mathbf{V}_{E}^{0}\right)^{H} \mathbf{R}_{l k}^{0} \mathbf{V}_{E}^{0}, l=0,1, \cdots, L, k=1,2, \cdots, K$, and $\widetilde{\mathbf{n}}_{\text {null }} \sim \mathcal{C N}\left(0, \tau N_{0} \mathbf{I}_{M}\right)$. Then, we estimate $\mathbf{h}_{0 m, \text { null }}^{0}$ as

$$
\begin{aligned}
& \widehat{\mathbf{h}}_{0 m, \text { null }}^{0}= \\
& \sqrt{P_{0 m}} \mathbf{R}_{0 m, \text { null }}^{0}\left(N_{0} \mathbf{I}_{M}+\tau \sum_{t=0}^{L} P_{t m} \mathbf{R}_{t m, \text { null }}^{0}\right)^{-1} \widetilde{\mathbf{y}}_{0 m, \text { null }},
\end{aligned}
$$

where

$$
\begin{aligned}
\widetilde{\mathbf{y}}_{0 m, \text { null }}= & \sqrt{P_{0 m}} \tau \mathbf{h}_{0 m, \text { null }}^{0}+\sum_{t=1}^{L} \sqrt{P_{t m}} \tau \mathbf{h}_{t m, \text { null }}^{0} \\
& +\sqrt{P_{E}} \tau\left(\mathbf{V}_{E}^{0}\right)^{H} \mathbf{h}_{E}^{0}+\left(\boldsymbol{\omega}_{m} \otimes \mathbf{I}_{M}\right)^{H} \widetilde{\mathbf{n}}_{\text {null }} .
\end{aligned}
$$

Similarly, we have $\mathbf{h}_{0 m, \text { null }}^{0}=\widehat{\mathbf{h}}_{0 m, \text { null }}^{0}+\mathbf{e}_{0 m, \text { null }}^{0}$, where $\widehat{\mathbf{h}}_{0 m}^{0} \sim \mathcal{C N}\left(\mathbf{0}_{N_{t}}, \widehat{\mathbf{R}}_{0 m, \text { null }}^{0}\right)$ and $\mathbf{e}_{0 m, \text { null }}^{0} \sim$ $\mathcal{C N}\left(\mathbf{0}_{N_{t}}, \mathbf{R}_{0 m, \text { null }}^{0}-\widehat{\mathbf{R}}_{0 m, \text { null }}^{0}\right)$, with

$$
\begin{aligned}
& \widehat{\mathbf{R}}_{0 m, \text { null }}^{0}= \\
& P_{0 m} \tau \mathbf{R}_{0 m, \text { null }}^{0}\left(N_{0} \mathbf{I}_{M}+\tau \sum_{t=0}^{L} P_{t m} \mathbf{R}_{t m, \text { null }}^{0}\right)^{-1} \mathbf{R}_{0 m, \text { null }}^{0} .
\end{aligned}
$$

In the downlink data transmission phase, we employ the same transmission scheme as in Section II-B, but replace $\mathbf{w}_{0 m}$ with $\mathbf{w}_{0 m, \text { null }}=\mathbf{V}_{E}^{0} \frac{\widehat{\mathbf{h}}_{0 m, \text { null }}^{0}}{\left\|\widehat{\mathbf{h}}_{0 m, \text { null }}^{0}\right\|}$, and set $p=1 / K, q=0$. We refer to this transmission design as "Null Space Design". Following the same approach as in Appendix A, we can show that the asymptotic achievable secrecy rate of the "Null Space Design" is still given by (21) after replacing $\mathbf{R}_{0 m}$ and $\widehat{\mathbf{R}}_{0 m}^{0}$ with $\mathbf{R}_{0 m, \text { null }}$ and $\widehat{\mathbf{R}}_{0 m, \text { null }}^{0}$ in $\theta_{m}$, respectively, and setting $\mathbf{R}_{E}^{0}=\mathbf{0}$ in (21). The other terms in (21) remain unchanged.
Remark 3: The proposed null space design transmits the signal along the orthogonal subspace $\mathbf{V}_{E}^{0}$. As a result, the performance of the null space design depends on the rank of $\mathbf{V}_{E}^{0}$. For instance, for the extreme case of i.i.d. fading, $\mathbf{V}_{E}^{0}$ does not exist and hence the null space design is not applicable. In practice, the null space design will perform better in highly correlated channels, under strong pilot contamination attack, and in the high SNR regime since it can effectively degrade the eavesdropper's performance in these scenarios. Matched filter precoding and AN generation, in contrast, will perform better in weakly correlated channels, under weak pilot contamination attack, and in the low SNR regime. Based on the expression in (21), an analytical criterion can be obtained to determine whether matched filter precoding with AN generation or the null space design is preferable for a given channel and eavesdropper parameters.

\section{NumERICAL RESUltS}

In this section, we provide numerical results to evaluate the secrecy performance of the considered massive MIMO system with an active eavesdropper. We consider a system where a $N_{t}=128$ uniform linear array is employed at the base station, the antenna spacing is half a wavelength, and the angle of arrival (AoA) interval is $\mathcal{A}=[-\pi / 2, \pi / 2]$. We use the truncated Laplacian distribution to model the channel power angle spectrum as [18]

$$
p(\theta)=\frac{1}{\sqrt{2} \sigma\left(1-e^{-\sqrt{2} \pi / \sigma}\right)} e^{\frac{-\sqrt{2}\|\|_{\theta-\bar{\theta}} \|}{\sigma},}
$$

where $\sigma$ and $\bar{\theta}$ denote the angular spread (AS) and the mean AoA of the channel, respectively. We assume that the ASs $\sigma$ in (56) for the channels of all users and the eavesdropper are identical and we set $\sigma=\pi / 2$. The channel covariance matrices of all users and the eavesdropper are generated based on [18, Eq. (3.14)]. For the channel between the user and the BS in its own cell and the channel between the user and the BSs in the other cells, we impose a channel power normalization to make the trace of the channel covariance matrices equal to $N_{t}$ and $\rho N_{t}$, respectively, and set $\rho=0.1$. The asymptotic secrecy rate is computed from Proposition 1 and the exact secrecy rate is obtained by Monte Carlo simulation. We set $L=3$, $K=5, P_{l k}=1, k=1,2, \cdots, K, l=0,1, \cdots, L, \tau=10$, and $N_{0}=1$. The mean channel AoAs $\bar{\theta}$ of all users and the eavesdropper in (56) are generated at random and the channel AoAs $\theta$ of all users and the eavesdropper are distributed within the angle interval $[-\pi, \pi]$ based on (56).

Figure 1 shows the secrecy rate performance vs. the SNR $\gamma$ for matched filter precoding and AN generation and different $p$. We observe from Figure 1 that the asymptotic secrecy rate in Proposition 1 provides a good estimate for the exact secrecy rate. Also, we observe from Figure 1 that in the low SNR regime, allocating more power (larger $p$ ) to the transmit signal leads to a higher secrecy rate. However, as the SNR increases, the secrecy rate may be small if the transmit signal power is high, i.e., if the AN power is small. For example, for SNR $=2$ $\mathrm{dB}$, we find from Corollary 2 that $p<0.15$ is a necessary condition to guarantee reliable communication. Thus, for $p=$ 0.18 , a positive secrecy rate cannot be achieved, as confirmed by Figure 1.

Figure 2 shows the exact secrecy rate performance vs. the SNR for different $P_{E}$ and different designs. For matched filter precoding with AN generation, we obtain the optimal power allocation $p$ and $q$ based on Corollary 1. For naive matched filter precoding, we set $p=1 / K$ and do not generate AN. From Figure 2 we make the following observations: 1) Because of the pilot contamination attack, even if the transmitter is equipped with a large number of antennas, naive 


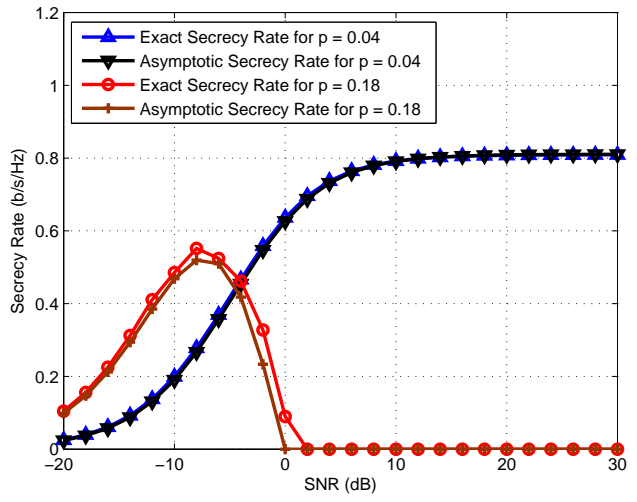

Fig. 1: Secrecy rate vs. the SNR for different $p$

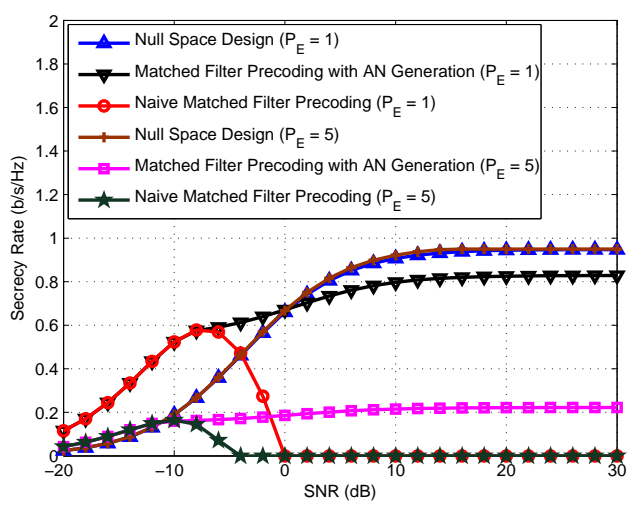

Fig. 2: Exact secrecy rate vs. the SNR for different $P_{E}$ and different precoding designs.

matched filter precoding can not achieve a positive secrecy rate for moderate-to-high SNR. 2) For $P_{E}=1$, matched filter precoding with AN generation performs better in the low-tomoderate SNR regime and the null space design performs better in the moderate-to-high SNR regime, see Remark 3. 3) When the eavesdropper increases the power of the pilot contamination attack, this results in a serious secrecy rate loss for matched filter precoding with AN generation, but has barely any visible impact on the null space design. This is because the null space design can eliminate the impact of pilot contamination caused by the active eavesdropper as suggest by (53) and significantly degrade the achievable rate of the active eavesdropper.

\section{Vi. CONClusions}

In this paper, we have studied the transmission design for multi-cell multi-user massive MIMO systems in the presence of an active eavesdropper. Assuming matched filter precoding and AN generation, we obtained an asymptotic achievable secrecy rate expression for the case of a pilot contamination attack when the number of transmit antennas tends to infinity. We obtained a closed-form expression for the optimal power allocation policy for the transmit signal and the AN. Moreover, we proved that the impact of the active eavesdropper is completely eliminated when the transmit correlation matrices of the users and the eavesdropper are orthogonal. This motivates the development of a null space design that exploits the low rank property of the transmit correlation matrices of massive
MIMO channels. Monte Carlo simulation results showed that the derived analytical results are accurate and confirmed the effectiveness of the proposed transmission schemes for combating the pilot contamination attack.

\section{APPENDIX A}

\section{PROOF OF PROPOSITION 1}

First, we calculate SINR $_{0 m}$ in (16). For the numerator of (16), based on [17, Corollary 1], we have

$$
\begin{aligned}
& \frac{1}{N_{t}}\left[\left|\left(\mathbf{h}_{0 m}^{0}\right)^{H} \mathbf{w}_{0 m}\right|^{2}\right] \\
& \stackrel{N_{t} \rightarrow \infty}{\rightarrow} \frac{1}{N_{t}}\left|\widehat{\mathbf{h}}_{0 m}^{0}\right|^{2}+\frac{1}{N_{t}}\left[\frac{\left(\widehat{\mathbf{h}}_{0 m}^{0}\right)^{H}\left(\mathbf{R}_{0 m}^{0}-\widehat{\mathbf{R}}_{0 m}^{0}\right) \widehat{\mathbf{h}}_{0 m}^{0}}{\left\|\widehat{\mathbf{h}}_{0 m}^{0}\right\|^{2}}\right] \\
& \stackrel{N_{t} \rightarrow \infty}{\rightarrow} \frac{1}{N_{t}} \operatorname{tr}\left(\widehat{\mathbf{R}}_{0 m}^{0}\right)+\frac{1}{N_{t}} \frac{\operatorname{tr}\left(\left(\mathbf{R}_{0 m}^{0}-\widehat{\mathbf{R}}_{0 m}^{0}\right) \widehat{\mathbf{R}}_{0 m}^{0}\right)}{\operatorname{tr}\left(\widehat{\mathbf{R}}_{0 m}^{0}\right)} .
\end{aligned}
$$

For the denominator of (16), we have

$$
\begin{aligned}
\frac{1}{N_{t}}\left|\left(\mathbf{h}_{0 m}^{0}\right)^{H} \mathbf{w}_{0 k}\right|^{2} & =\frac{\left(\mathbf{h}_{0 m}^{0}\right)^{H} \widehat{\mathbf{h}}_{0 k}^{0}\left(\widehat{\mathbf{h}}_{0 k}^{0}\right)^{H}\left(\mathbf{h}_{0 m}^{0}\right)}{\left(\widehat{\mathbf{h}}_{0 k}^{0}\right)^{H} \widehat{\mathbf{h}}_{0 k}^{0}} \\
& { }_{N_{t} \rightarrow \infty}^{\rightarrow} \frac{\left(\widehat{\mathbf{h}}_{0 k}^{0}\right)^{H} \mathbf{R}_{0 m}^{0} \widehat{\mathbf{h}}_{0 k}^{0}}{\left(\widehat{\mathbf{h}}_{0 k}^{0}\right)^{H} \widehat{\mathbf{h}}_{0 k}^{0}} \\
& \stackrel{N_{t} \rightarrow \infty}{\rightarrow} \frac{1}{N_{t}} \frac{\operatorname{tr}\left(\mathbf{R}_{0 m}^{0} \widehat{\mathbf{R}}_{0 k}^{0}\right)}{\operatorname{tr}\left(\widehat{\mathbf{R}}_{0 k}^{0}\right)} .
\end{aligned}
$$

Also, performing some simplifications, we have

$$
\begin{aligned}
& \frac{1}{N_{t}}\left[\left\|\left(\mathbf{h}_{0 m}^{0}\right)^{H} \mathbf{U}_{\text {null, }}\right\|^{2}\right]^{N_{t} \rightarrow \infty} \rightarrow \frac{1}{N_{t}} \mathbf{R}_{0 m}^{0} \\
& -\frac{1}{N_{t}} \sum_{k=1, k \neq m}^{K} \operatorname{tr}\left(\widehat{\mathbf{R}}_{0 k}^{0}\right)^{-1}\left(\mathbf{h}_{0 m}^{0}\right)^{H}\left(\widehat{\mathbf{h}}_{0 k}^{0}\right)\left(\widehat{\mathbf{h}}_{0 k}^{0}\right)^{H} \mathbf{h}_{0 m}^{0} \\
& -\frac{1}{N_{t}} \operatorname{tr}\left(\widehat{\mathbf{R}}_{0 m}^{0}\right)^{-1}\left(\mathbf{h}_{0 m}^{0}\right)^{H}\left(\widehat{\mathbf{h}}_{0 m}^{0}\right)\left(\widehat{\mathbf{h}}_{0 m}^{0}\right)^{H} \mathbf{h}_{0 m}^{0} .
\end{aligned}
$$

For $m \neq k, \mathbf{h}_{0 m}^{0}$ is independent of $\widehat{\mathbf{h}}_{0 k}^{0}$. Hence, the asymptotic expression for $\frac{1}{N_{t}}\left(\mathbf{h}_{0 m}^{0}\right)^{H} \widehat{\mathbf{h}}_{0 k}^{0}\left(\widehat{\mathbf{h}}_{0 k}^{0}\right)^{H} \mathbf{h}_{0 m}^{0}$ is given by the numerator of (61).

For $m=k$, based on (2), we have

$$
\begin{aligned}
\widehat{\mathbf{h}}_{0 m}^{0} & =\sqrt{P_{0 m}} \tau \mathbf{C}_{0 m}^{0} \mathbf{h}_{0 m}^{0}+\mathbf{C}_{0 m}^{0} \sum_{l=1}^{L} \sqrt{P_{l m}} \tau \mathbf{h}_{l m}^{0} \\
& +\mathbf{C}_{0 m}^{0} \sqrt{P_{E}} \tau \mathbf{h}_{E}^{0}+\mathbf{C}_{0 m}^{0} \boldsymbol{\Omega}_{m} \mathbf{n},
\end{aligned}
$$

where $\mathbf{C}_{0 m}^{0}$ is defined in (48) and $\boldsymbol{\Omega}_{m}=\left(\boldsymbol{\omega}_{m} \otimes \mathbf{I}_{N_{t}}\right)^{H}$.

Based on (63), we obtain

$$
\begin{aligned}
& \left(\mathbf{h}_{0 m}^{0}\right)^{H} \widehat{\mathbf{h}}_{0 m}^{0}\left(\widehat{\mathbf{h}}_{0 m}^{0}\right)^{H} \mathbf{h}_{0 m}^{0}=\left(\mathbf{h}_{0 m}^{0}\right)^{H} \mathbf{C}_{0 m}^{0} \boldsymbol{\Omega}_{m} \mathbf{n}\left(\widehat{\mathbf{h}}_{0 m}^{0}\right)^{H} \mathbf{h}_{0 m}^{0} \\
& +\tau^{2}\left(\mathbf{h}_{0 m}^{0}\right)^{H} \mathbf{C}_{0 m}^{0} \sum_{t=0}^{L} \sum_{s=0}^{L} \sqrt{P_{t m}} \sqrt{P_{s m}} \mathbf{h}_{t m}^{0}\left(\mathbf{h}_{s m}^{0}\right)^{H}\left(\mathbf{C}_{0 m}^{0}\right)^{H} \mathbf{h}_{0 m}^{0} \\
& +\tau^{2} \sqrt{P_{E}}\left(\mathbf{h}_{0 m}^{0}\right)^{H} \mathbf{C}_{0 m}^{0} \sum_{t=0}^{L} \sqrt{P_{t m}} \mathbf{h}_{t m}^{0}\left(\mathbf{h}_{E}^{0}\right)^{H}\left(\mathbf{C}_{0 m}^{0}\right)^{H} \mathbf{h}_{0 m}^{0} \\
& +\tau\left(\mathbf{h}_{0 m}^{0}\right)^{H} \mathbf{C}_{0 m}^{0} \sum_{t=0}^{L} \sqrt{P_{t m}} \mathbf{h}_{t m}^{0} \mathbf{h}^{H} \boldsymbol{\Omega}_{m}^{H}\left(\mathbf{C}_{0 m}^{0}\right)^{H} \mathbf{h}_{0 m}^{0} \\
& +\tau \sqrt{P_{E}}\left(\mathbf{h}_{0 m}^{0}\right)^{H} \mathbf{C}_{0 m}^{0} \mathbf{h}_{E}^{l}\left(\widehat{\mathbf{h}}_{0 m}^{0}\right)^{H} \mathbf{h}_{0 m}^{0}
\end{aligned}
$$


When $N_{t} \rightarrow \infty$, based on (64) and [17, Corollary 1], we have

$$
\begin{aligned}
& \frac{1}{N_{t}}\left(\mathbf{h}_{0 m}^{0}\right)^{H} \widehat{\mathbf{h}}_{0 m}^{0}\left(\widehat{\mathbf{h}}_{0 m}^{0}\right)^{H} \mathbf{h}_{0 m}^{0} \\
& \stackrel{N_{t} \rightarrow{ }^{\infty}}{\rightarrow} \frac{1}{N_{t}} \tau^{2}\left(\mathbf{h}_{0 m}^{0}\right)^{H} \mathbf{C}_{0 m}^{0} \sum_{t=0}^{L} P_{t m} \mathbf{h}_{t m}^{0}\left(\mathbf{h}_{t m}^{0}\right)^{H}\left(\mathbf{C}_{0 m}^{0}\right)^{H} \mathbf{h}_{0 m}^{0} \\
& +\frac{1}{N_{t}} \tau^{2} P_{E}\left(\mathbf{h}_{0 m}^{0}\right)^{H} \mathbf{C}_{0 m}^{0} \mathbf{h}_{E}^{0}\left(\mathbf{h}_{E}^{0}\right)^{H}\left(\mathbf{C}_{0 m}^{0}\right)^{H} \mathbf{h}_{0 m}^{0} \\
& +\frac{1}{N_{t}}\left(\mathbf{h}_{0 m}^{0}\right)^{H} \mathbf{C}_{0 m}^{0} \boldsymbol{\Omega}_{m} \mathbf{n}\left(\mathbf{C}_{0 m}^{0} \boldsymbol{\Omega}_{m} \mathbf{n}\right)^{H} \mathbf{h}_{0 m}^{0} \\
& =\frac{1}{N_{t}} \tau^{2} P_{0 m}\left(\mathbf{h}_{0 m}^{0}\right)^{H} \mathbf{C}_{0 m}^{0} \mathbf{h}_{0 m}^{0}\left(\mathbf{h}_{0 m}^{0}\right)^{H}\left(\mathbf{C}_{0 m}^{0}\right)^{H} \mathbf{h}_{0 m}^{0} \\
& +\frac{1}{N_{t}} \tau^{2}\left(\mathbf{h}_{0 m}^{0}\right)^{H} \mathbf{C}_{0 m}^{0} \sum_{t=1}^{L} P_{t m} \mathbf{h}_{t m}^{0}\left(\mathbf{h}_{t m}^{0}\right)^{H}\left(\mathbf{C}_{0 m}^{0}\right)^{H} \mathbf{h}_{0 m}^{0} \\
& +\frac{1}{N_{t}} \tau^{2} P_{E}\left(\mathbf{h}_{0 m}^{0}\right)^{H} \mathbf{C}_{0 m}^{0} \mathbf{h}_{E}^{0}\left(\mathbf{h}_{E}^{0}\right)^{H}\left(\mathbf{C}_{0 m}^{0}\right)^{H} \mathbf{h}_{0 m}^{0} \\
& +\frac{1}{N_{t}}\left(\mathbf{h}_{0 m}^{0}\right)^{H} \mathbf{C}_{0 m}^{0} \boldsymbol{\Omega}_{m} \mathbf{n}\left(\mathbf{C}_{0 m}^{0} \boldsymbol{\Omega}_{m} \mathbf{n}\right)^{H} \mathbf{h}_{0 m}^{0}{ }^{N_{t} \rightarrow \infty} \rightarrow \frac{1}{N_{t}} \Lambda_{0 m}^{0} .
\end{aligned}
$$

Also, for $m \neq k, \mathbf{h}_{0 m}^{l}$ is independent of $\widehat{\mathbf{h}}_{l k}^{l}$, and we obtain in (17)

$$
\begin{aligned}
\frac{1}{N_{t}}\left|\left(\mathbf{h}_{0 m}^{l}\right)^{H} \mathbf{w}_{l k}\right|^{2} & =\frac{1}{N_{t}}\left(\mathbf{h}_{0 m}^{l}\right)^{H} \frac{\widehat{\mathbf{h}}_{l k}^{l}}{\left|\widehat{\mathbf{h}}_{l k}^{l}\right|} \frac{\left(\widehat{\mathbf{h}}_{l k}^{l}\right)^{H}}{\left|\widehat{\mathbf{h}}_{l k}^{l}\right|} \mathbf{h}_{0 m}^{l} \\
& \stackrel{N_{t} \rightarrow \infty}{\rightarrow} \frac{\left(\widehat{\mathbf{h}}_{l k}^{l}\right)^{H} \mathbf{R}_{0 m}^{l} \widehat{\mathbf{h}}_{l k}^{l}}{\left|\widehat{\mathbf{h}}_{l k}^{l}\right|^{2}} \stackrel{N_{t} \rightarrow \infty}{\rightarrow} \frac{\operatorname{tr}\left(\mathbf{R}_{0 m}^{l} \widehat{\mathbf{R}}_{l k}^{l}\right)}{\operatorname{tr}\left(\widehat{\mathbf{R}}_{l k}^{l}\right)} .
\end{aligned}
$$

For $m=k$, similar to (65), we have

$$
\begin{aligned}
\frac{1}{N_{t}}\left|\left(\mathbf{h}_{0 m}^{l}\right)^{H} \mathbf{w}_{l m}\right|^{2} & =\frac{1}{N_{t}} \frac{\left(\mathbf{h}_{0 m}^{l}\right)^{H} \widehat{\mathbf{h}}_{l m}^{l}\left(\widehat{\mathbf{h}}_{l m}^{l}\right)^{H} \mathbf{h}_{0 m}^{l}}{\left|\widehat{\mathbf{h}}_{l m}^{l}\right|^{2}} \\
& \stackrel{N_{t} \rightarrow \infty}{\rightarrow} \frac{1}{N_{t}} \frac{\Lambda_{0 m}^{l}}{\operatorname{tr}\left(\widehat{\mathbf{R}}_{l m}^{l}\right)} .
\end{aligned}
$$

Next, we simplify

$$
\begin{aligned}
& \left(\mathbf{h}_{0 m}^{l}\right)^{H} \mathbf{U}_{\mathrm{null}, l} \mathbf{U}_{\mathrm{null}, l}^{H} \mathbf{h}_{0 m}^{l}=\operatorname{tr}\left(\mathbf{R}_{0 m}^{l}\right)- \\
& \sum_{k=1, k \neq m}^{K} \operatorname{tr}\left(\widehat{\mathbf{R}}_{l k}^{l}\right)^{-1}\left(\mathbf{h}_{0 m}^{l}\right)^{H}\left(\widehat{\mathbf{h}}_{l k}^{l}\right)\left(\widehat{\mathbf{h}}_{l k}^{l}\right)^{H} \mathbf{h}_{0 m}^{l} \\
& -\operatorname{tr}\left(\widehat{\mathbf{R}}_{l m}^{l}\right)^{-1}\left(\mathbf{h}_{0 m}^{l}\right)^{H}\left(\widehat{\mathbf{h}}_{l m}^{l}\right)\left(\widehat{\mathbf{h}}_{l m}^{l}\right)^{H} \mathbf{h}_{0 m}^{l} .
\end{aligned}
$$

Following a similar approach as was used to obtain (61) and (65), we obtain

$$
\begin{aligned}
& \frac{1}{N_{t}}\left(\mathbf{h}_{0 m}^{l}\right)^{H}\left(\widehat{\mathbf{h}}_{l k}^{l}\right)\left(\widehat{\mathbf{h}}_{l k}^{l}\right)^{H} \mathbf{h}_{0 m}^{l} \stackrel{N_{t} \rightarrow \infty}{\rightarrow} \operatorname{tr}\left(\mathbf{R}_{0 m}^{l} \widehat{\mathbf{R}}_{l k}^{l}\right) \\
& \frac{1}{N_{t}}\left(\mathbf{h}_{0 m}^{l}\right)^{H}\left(\widehat{\mathbf{h}}_{l m}^{l}\right)\left(\widehat{\mathbf{h}}_{l m}^{l}\right)^{H} \mathbf{h}_{0 m}^{l} \stackrel{N_{t} \rightarrow \infty}{\rightarrow} \Lambda_{0 m}^{l} .
\end{aligned}
$$

By substituting (57)-(72) into (16), we obtain the expression for $\mathrm{SINR}_{0 m}$, asy in (22). Next, we simplify $\mathrm{SINR}_{\text {eve }}$ in (19). First, we consider

$$
\left(\mathbf{h}_{E}^{0}\right)^{H} \mathbf{w}_{0 m} \mathbf{w}_{0 m}^{H} \mathbf{h}_{E}^{0}=\frac{\left(\mathbf{h}_{E}^{0}\right)^{H} \widehat{\mathbf{h}}_{0 m}^{0}\left(\widehat{\mathbf{h}}_{0 m}^{0}\right)^{H} \mathbf{h}_{E}^{0}}{\left|\widehat{\mathbf{h}}_{0 m}^{0}\right|^{2}} .
$$

When $N_{t} \rightarrow \infty$, we have

$$
\begin{aligned}
& \frac{1}{N_{t}}\left(\mathbf{h}_{E}^{0}\right)^{H} \widehat{\mathbf{h}}_{0 m}^{0}\left(\widehat{\mathbf{h}}_{0 m}^{0}\right)^{H} \mathbf{h}_{E}^{0} \stackrel{N_{t} \rightarrow \infty}{\rightarrow} \frac{1}{N_{t}} \Lambda_{E}^{0} \\
& \frac{1}{N_{t}}\left|\widehat{\mathbf{h}}_{0 m}^{0}\right|^{2} \stackrel{N_{t} \rightarrow \infty}{\rightarrow} \frac{1}{N_{t}} \operatorname{tr}\left(\widehat{\mathbf{R}}_{0 m}^{0}\right) .
\end{aligned}
$$

$\frac{1}{N_{t}}\left(\mathbf{h}_{E}^{l}\right)^{H} \mathbf{U}_{\text {null }, l} \mathbf{U}_{\text {null }, l}^{H} \mathbf{h}_{E}^{l}$ can be expressed as

$$
\frac{1}{N_{t}}\left(\mathbf{h}_{E}^{l}\right)^{H} \mathbf{U}_{\mathrm{null}, l} \mathbf{U}_{\mathrm{null}, l}^{H} \mathbf{h}_{E}^{l}
$$$$
=\frac{1}{N_{t}} \operatorname{tr}\left(\mathbf{R}_{E}^{l}\right)-\sum_{k=1, k \neq m}^{K} \operatorname{tr}\left(\widehat{\mathbf{R}}_{l k}^{l}\right)^{-1} \frac{1}{N_{t}}\left(\mathbf{h}_{E}^{l}\right)^{H}\left(\widehat{\mathbf{h}}_{l k}^{l}\right)\left(\widehat{\mathbf{h}}_{l k}^{l}\right)^{H} \mathbf{h}_{E}^{l}
$$$$
-\operatorname{tr}\left(\widehat{\mathbf{R}}_{l k}^{l}\right)^{-1} \frac{1}{N_{t}}\left(\mathbf{h}_{E}^{l}\right)^{H}\left(\widehat{\mathbf{h}}_{l m}^{l}\right)\left(\widehat{\mathbf{h}}_{l m}^{l}\right)^{H} \mathbf{h}_{E}^{l} .
$$

The asymptotic expressions for $\frac{1}{N_{t}}\left(\mathbf{h}_{E}^{l}\right)^{H}\left(\widehat{\mathbf{h}}_{l k}^{l}\right)\left(\widehat{\mathbf{h}}_{l k}^{l}\right)^{H} \mathbf{h}_{E}^{l}$ and $\frac{1}{N_{t}}\left(\mathbf{h}_{E}^{l}\right)^{H}\left(\widehat{\mathbf{h}}_{l m}^{l}\right)\left(\widehat{\mathbf{h}}_{l m}^{l}\right)^{H} \mathbf{h}_{E}^{l}$ in (76) are given by

$$
\frac{1}{N_{t}}\left(\mathbf{h}_{E}^{l}\right)^{H} \widehat{\mathbf{h}}_{l k}^{l}\left(\widehat{\mathbf{h}}_{l k}^{l}\right)^{H} \mathbf{h}_{E}^{l} \stackrel{N_{t} \rightarrow \infty}{\rightarrow} \frac{1}{N_{t}} \operatorname{tr}\left(\mathbf{R}_{E}^{l} \widehat{\mathbf{R}}_{l k}^{l}\right)
$$

and

respectively.

$$
\frac{1}{N_{t}}\left(\mathbf{h}_{E}^{l}\right)^{H} \widehat{\mathbf{h}}_{l m}^{l}\left(\widehat{\mathbf{h}}_{l m}^{l}\right)^{H} \mathbf{h}_{E}^{l} \stackrel{N_{t} \rightarrow \infty}{\rightarrow} \frac{1}{N_{t}} \Lambda_{E}^{l},
$$

Substituting (73)-(78) into (19), we obtain (23), which completes the proof.

\section{REFERENCES}

[1] A. D. Wyner, "The wiretap channel," Bell Syst. Tech. J., vol. 54, pp. 1355-1387, Oct. 1975

[2] A. Khisti and G. W. Wornell, "Secure transmission with multiple antennas-Part II: The MIMOME wiretap channel," IEEE Trans. Inf. Theory, vol. 56, pp. 5515-5532, Nov. 2010.

[3] F. Oggier and B. Hassibi, "The secrecy capacity of the MIMO wiretap channel," IEEE Trans. Inf. Theory, vol. 57, pp. 4961-4972, Aug. 2011.

[4] Y. Wu, C. Xiao, Z. Ding, X. Gao, and S. Jin, "Linear precoding for finite alphabet signaling over MIMOME wiretap channels," IEEE Trans. Veh. Technol., vol. 61, pp. 2599-2612, Jul. 2012.

[5] S. Goel and R. Negi, "Guaranteeing secrecy using artificial noise," IEEE Trans. Wireless Commun., vol. 6, pp. 2180-2189, Jun. 2008.

[6] X. Zhou, B. Maham, and A. Hjørungnes, "Pilot contamination for active eavesdropping," IEEE Trans. Wireless Commun., vol. 11, pp. 903-907, Mar. 2012.

[7] T. L. Marzetta, "Noncooperative cellular wireless with unlimited numbers of base station antennas," IEEE Trans. Wireless Commun., vol. 9, pp. 3590-3600, Nov. 2010.

[8] J. Jose, A. Ashikhmin, T. L. Marzetta, and S. Vishwanath, "Pilot contamination and precoding in multi-cell TDD systems," IEEE Trans. Wireless Commun., vol. 10, pp. 2640-2651, Aug. 2011.

[9] H. Yin, D. Gesbert, M. Filippou, and Y. Liu, "A coordinated approach to channel estimation in large-scale multiple-antenna systems," IEEE $J$ Sel. Areas Commun., vol. 31, pp. 264-273, Feb. 2013.

[10] A. Adhikary, J. Nam, J.-Y. Ahn, and G. Caire, "Joint spatial division and multiplexing-The large-scale array regime," IEEE Trans. Inf. Theory, vol. 59, pp. 6441-6463, Oct. 2013.

[11] Y. Wu, C.-K. Wen, C. Xiao, X. Gao, and R. Schober, "Linear precoding for the MIMO multiple access channel with finite alphabet inputs and statistical CSI," to appear in IEEE Trans. Wireless Commun..

[12] X. Chen, J. Chen, and T. Liu, "Secure wireless information and power transfer in large-scale MIMO relaying systems with imperfect CSI," [Online]. Available: http://arxiv.org/abs/1407.5355v1.pdf, Jul. 2014.

[13] J. Zhu, R. Schober, and V. K. Bhargava, "Secure transmission in multicell massive MIMO systems," IEEE Trans. Wireless Commun., vol. 13, pp. 4766-4781, Sep. 2014.

[14] S. Im, H. Jeon, J. Choi, and J. Ha, "Secret key agreement under an active attack in MU-TDD systems with large antenna arrays," in Proc. IEEE Global. Telecommun. Conf. (GLOBECOM 2013), Atlanta, USA, Dec. 2013, pp. 1849-1855.

[15] C.-K. Wen, G. Pan, K.-K. Wong, M. Guo, and J.-C. Chen, "A deterministic equivalent for the analysis of non-Gaussian correlated MIMO multiple access channels," IEEE Trans. Inf. Theory, vol. 59, pp. 329352, Jan. 2013

[16] T. Kailath, A. H. Sayed, and B. Hassibi, Linear Estimation. New Jersey: Prentice Hall, 2000.

[17] J. Evans and D. N. C. Tse, "Large system performance of linear multiuser receivers in multipath fading channels," IEEE Trans. Inf. Theory, vol. 46, pp. 2059-2078, Sep. 2000.

[18] Y. S. Cho, J. Kim, W. Y. Yang, and C. G. Kang, MIMO-OFDM Wireless Communications with MATLAB. Singapore: John Wiley \& Sons (Asia) Pte Ltd, 2010. 